Original Article

\title{
Differences in the total hip rotation range of motion between sides in healthy young Japanese adults
}

\author{
Heonsoo Han, PT, PhD ${ }^{1)^{*}}$, Akira Kubo, PT, PhD ${ }^{1)}$, Masahiro Ishizaka, PT, PhD ${ }^{1)}$, \\ Hitoshi Maruyama, PT, $\mathrm{PhD}^{2)}$ \\ 1) Department of Physical Therapy, International University of Health and Welfare: 2600-1 Kitakane- \\ maru, Otawara, Tochigi 324-8501, Japan \\ 2) Fukuoka International University of Health and Welfare, Japan
}

\begin{abstract}
Purpose] To investigate the difference by degrees in total hip rotation range of motion (ROM) between sides and with regard to gender in healthy young Japanese adults. [Participants and Methods] Data from previously published studies were used. We utilized the left and right hip rotation ROM of 205 Japanese participants aged 18 to 25 years, who satisfied the inclusion criteria and were randomly measured three times in the prone position by the double-blind method. The average value of the three measurements was used to calculate the left-right difference of the hip total rotation ROM in the hip joints, which is the sum of the hip internal and external rotation ROM. We investigated the distribution of the total hip rotation considering the flexibility factor and its relationship with gender. [Results] We confirmed that there were seven participants with 2 SD or more and two with 3 SD or more. No significant difference was found in the left-right difference in the total hip rotation ROM, regardless of gender. [Conclusion] The distribution of left-right differences in the total hip rotation ROM in healthy young adults showed the existence of a significant left-right difference of 2 SD or more.

Key words: Total hip rotation, Range of motion, Laterality
\end{abstract}

(This article was submitted Aug. 19, 2020, and was accepted Oct. 25, 2020)

\section{INTRODUCTION}

Evaluation of the left-right difference in hip rotation range of motion (ROM) and imbalance of internal/external rotation is used in the clinical setting of physical therapy for low back pain and knee pain. Studies related to hip rotation ROM have been reported in a wide variety of areas such as low back pain ${ }^{1)}$, knee pain $^{2,3)}$, scoliosis $^{4)}$, sports field ${ }^{5,6}$ ).

In such a wide variety of studies that refer to total hip rotation (ToHiRo) ROM, which is the sum of the internal and external rotation ROM of the hip joint, in patients with patellofemoral pain, the left and right ToHiRo ROMs were compared between patellofemoral pain patients and healthy persons, and the left and right data regarding ToHiRo are shown but the distribution of the left-right difference were not shown ${ }^{3)}$. A study about judoka ${ }^{5)}$ reported that judoka athletes with low back pain have less ToHiRo than those with no low back pain. In a previously published study on hip joint rotation ROM in the sitting and prone measurement positions ${ }^{7)}$, ToHiRo was presented as the mean and standard deviation. In a study on ipsilateral hip rotation $\mathrm{ROM}^{8)}$, the balance of internal rotation and external rotation on one side was classified into patterns and analyzed. In this study, we focused on the distribution of the degree of the left-right difference of the ToHiRo ROM in consideration of flexibility in hip joint rotation in healthy participants. This is because even if a healthy person has a significant left-right difference, there is some possibility of obtaining data on other physical characteristics and motor functions even without symptoms such as pain.

*Corresponding author. Heonsoo Han (E-mail: han@iuhw.ac.jp)

(C2021 The Society of Physical Therapy Science. Published by IPEC Inc.

(c) (1) $\odot$ This is an open-access article distributed under the terms of the Creative Commons Attribution Non-Commercial No Deriva-

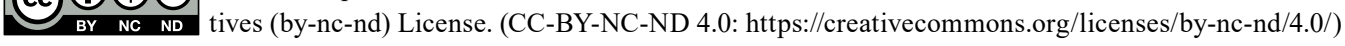


The distribution of left-right differences in ToHiRo ROM considering flexibility in healthy Japanese individuals is unknown. It was hypothesized that the comparison of ToHiRo ROMs between the left and right sides of healthy participants would reveal ToHiRo ROMs that are equal or close to equal and ToHiRo ROMs that are not equal.

The purpose of this study was to investigate the distribution of ToHiRo ROM according to the degree of the difference between the sides and gender differences in healthy participants. By conducting a fact-finding survey on the left-right difference in the ToHiRo ROM in healthy individuals, we obtained basic information on the degree of left-right difference and normality of the total hip ROM. We believe that the significant left-right difference in the ToHiRo ROM of healthy people will be an important fact when pursuing future relationships with physical characteristics and motor functions.

\section{PARTICIPANTS AND METHODS}

The participants that met the inclusion criteria were 205 healthy Japanese students (148 males and 57 females) enrolled in medical colleges (Table 1). The inclusion criteria were participants aged 18 to 25 years ${ }^{9)}$ and those who were not being treated for orthopedic diseases or who were able to walk independently without their daily lives being affected. Exclusion criteria were those who were pregnant, those who were currently undergoing treatment for paralysis/fractures in the pelvis and lower limbs, arthritis, and sprains, and those who had a history of surgery or a diagnosis of a hip joint disease.

Twenty-four of 229 people met the exclusion criteria: 21 were aged $\geq 25$ years, two had a medical history of hip joint diseases, and one was an outlier in hip joint rotation ROM examined by the Grubbs-Smirnov test. Measurements of hip rotation ROM were used in the analysis despite the lack of characteristic data.

Participants were provided with written or verbal approval prior to participation. Participants were told that they could stop the measurement during the course of being measured if they wanted and that they could withdraw without any repercussions after the measurement. This study was approved by the International University of Health and Welfare Ethics Review Board (approval number: 18-Io-42).

The measurement of the left and right hip joint rotation ROM was performed passively after the measurement order was randomly determined by drawing in advance. The measurement was performed thrice in a prone position using a metal goniometer (University of Tokyo type); for better precision, the ROM was measured using a scale with 1-degree intervals ${ }^{8)}$. The average value of the three measurements was used. The measurement position was the prone position because it is close to the joint angle assumed during the daily standing and walking motions. The pelvis was fixed by holding it down by hand so that it would not float when measuring the rotation ROMs of the hip joint in the prone position. The method of manually fixing the pelvis requires fixing the pelvis, fixing the hip joint by moving it to the final zone, and applying a goniometer at the same time. It is, therefore, difficult to be measured by one person, even an experienced physical therapist.

In order to improve the measurement accuracy and obtain objective data, the measurement process was divided, and multiple people were responsible for different roles. The roles were divided into four parts: a person in charge of movement who was required to move the limb to the end of the hip rotation ROM, a person who applies the goniometer, a person in charge of reading and recording scales of the goniometer, and observers to monitor whether or not the measurement was correctly performed. Since the measured values were not shared until the end of the measurement. We ensured double-blinding - the participants and those who measured the values did not share the measurement values with each other.

Before the measurement, a physical therapist instructed the students about the measurement method. The physical therapist, with $\geq 7$ years of experience supervised the students throughout the measurement process and performed part of the measurement at the same time.

When measuring the ROM of the joint, the hip joint on the side being measured was placed in the intermediate position of adduction and abduction. The knee position was then flexed at $90^{\circ}$, and measuring was started. When the contralateral lower extremity restricted the final region during the measurement of external rotation, the contralateral lower limb was abducted.

In the data analysis, ToHiRo ROM was calculated on the left and right sides. The sum of the left and right ToHiRo ROMs was used to calculate the ratio (\%) of the ToHiRo ROM of the left or right side to the sum of ToHiRo, and the difference from $50 \%$ was calculated. This indicates that the larger the difference from $50 \%$, the larger the left-right difference. The difference from $50 \%$ on each of the left and right sides was calculated as the frequency distribution and proportion for each class. If the

Table 1. Characteristics of participants

\begin{tabular}{lccc}
\hline & Male & Female & Total \\
& $\mathrm{n}=148$ & $\mathrm{n}=57$ & $\mathrm{n}=205$ \\
\hline Age (years) & $20.2 \pm 1.1$ & $20.0 \pm 1.1$ & $20.1 \pm 1.1$ \\
Height $(\mathrm{cm})$ & $170.9 \pm 5.8$ & $158.2 \pm 5.7^{*_{1}}$ & $167.4 \pm 8.1^{*_{3}}$ \\
Weight $(\mathrm{kg})$ & $62.2 \pm 9.4$ & $54.2 \pm 10.1^{*_{2}}$ & $60.1 \pm 10.2^{* 4}$ \\
BMI $\left(\mathrm{kg} / \mathrm{m}^{2}\right)$ & $21.3 \pm 2.8$ & $21.6 \pm 3.7^{* 2}$ & $21.4 \pm 3.1^{* 4}$ \\
\hline
\end{tabular}

Mean $\pm \mathrm{SD}$

${ }^{{ }_{1}} \mathrm{n}=56,{ }^{* 2} \mathrm{n}=54,{ }^{* 3} \mathrm{n}=204,{ }^{* 4} \mathrm{n}=202$. 
proportion of left hip total rotation on sum total rotation (\%) is less than $50 \%$, the ToHiRo on the right side is larger than that on the left side; if it is $50 \%$, there is no left-right difference; and if it is over $50 \%$, the left side is larger.

The class setting in the creation of the table showing the distribution of each analysis value was such that all minimum and maximum values were included. For the class in the table, the distribution of the ToHiRo ROM on the left and right sides was $10^{\circ}$. The class interval for the "distribution of the proportion (\%) of the ToHiRo ROM on the side where the ToHiRo ROM was greater divided by the ToHiRo ROM sum" was $1 \%$. The greater side of the ToHiRo ROM classified by standard deviation was set to $1 \mathrm{SD}$ unit. For the unit of distribution of "the greater side of ToHiRo ROM," the standard deviation was used to express the distance from the center value for convenience.

The ratio of left and right ToHiRo to "ToHiRo ROM sum" was calculated, and the difference from $50 \%$ was used to express the larger side and the degree of the left-right difference. Subsequently, a frequency distribution table was created by classifying the standard deviation for each gender. The normality test using the Shapiro-Wilk test was performed in the left ToHiRo, right ToHiRo, ToHiRo ROM sum, and "proportion of left ToHiRo on ToHiRo ROM sum".

An unpaired t-test was performed to examine the gender difference in four items: each of internal rotation and external rotation, ToHiRo, and "ToHiRo ROM sum". In the left-right difference of ToHiRo ROM, the larger side of ToHiRo was aggregated separately for left and right, and it was examined using the $\chi^{2}$ test of independence to confirm whether it was independent of the appearance frequency of males and females ${ }^{10)}$.

In the $\chi^{2}$ test of independence, the null hypothesis $\mathrm{H}_{0}$ was determined to be independent of the two factors, and the alternative hypothesis $\mathrm{H}_{1}$ was determined assuming that the two factors were not independent (that is, related) at a significance level of $5 \%$.

\section{RESULTS}

The basic data on the left and right hip joint ROM, ToHiRo of both sides, ToHiRo ROM sum, and proportion (\%) of left ToHiRo of ToHiRo ROM sum are shown in Table 2. In the comparison of basic data between males and females, a significant difference was found in the left and right internal and external rotation ROMs, but no significant difference was found in the remaining four items. In the range of "proportion (\%) of left ToHiRo of ToHiRo ROM sum", the minimum value was $44.5 \%$ with the left side $5.5 \%$ smaller and the maximum value was $56.1 \%$ with the left side $6.1 \%$ larger.

In the test of normality in the four items of left ToHiRo, right ToHiRo, ToHiRo ROM sum, and "proportion (\%) of left ToHiRo of ToHiRo ROM sum", all items showed a normal distribution with a significant probability of $\mathrm{p}>0.05$. The ROMs for ToHiRo on the left and right sides were distributed from 50-59 to 150-159 (Table 3). The relative frequency of the classes with ROMs closest to $90^{\circ}$ is the highest, and both left ToHiRo and right ToHiRo are normally distributed (left ToHiRo $\mathrm{p}=0.31$, right ToHiRo $\mathrm{p}=0.08$ ).

The proportion (\%) of the ToHiRo ROM to the ToHiRo ROM sum on each of the left and right sides was normally distributed ( $\mathrm{p}=0.17$ ) (Table 4). On examining, using the $\chi^{2}$ test of independence, whether the appearance ratio of the degree

Table 2. Hip rotation ROM and proportion of left ToHiRo of ToHiRo ROM sum (\%)

\begin{tabular}{|c|c|c|c|c|c|c|}
\hline & \multicolumn{2}{|c|}{ Male $(n=148)$} & \multicolumn{2}{|c|}{ Female $(n=57)$} & \multicolumn{2}{|c|}{ Total $(n=205)$} \\
\hline & Mean \pm SD & $\begin{array}{c}\text { Range } \\
\text { (Min-Max) }\end{array}$ & Mean \pm SD & $\begin{array}{c}\text { Range } \\
\text { (Min-Max) }\end{array}$ & Mean \pm SD & $\begin{array}{c}\text { Range } \\
\text { (Min-Max) }\end{array}$ \\
\hline Left IR $\left(^{\circ}\right)$ & $43.3 \pm 10.0 * *$ & $\begin{array}{c}59 \\
(16-75)\end{array}$ & $52.2 \pm 11.4^{* *}$ & $\begin{array}{c}48.6 \\
(22.7-71.3)\end{array}$ & $45.8 \pm 11.1$ & $\begin{array}{c}59 \\
(16-75)\end{array}$ \\
\hline Left ER $\left(^{\circ}\right)$ & $54.9 \pm 12.7^{*}$ & $\begin{array}{c}68 \\
(27-95)\end{array}$ & $50.6 \pm 11.4^{*}$ & $\begin{array}{c}42.7 \\
(27-69.7)\end{array}$ & $53.7 \pm 12.5$ & $\begin{array}{c}68 \\
(27-95)\end{array}$ \\
\hline Right IR $\left(^{\circ}\right)$ & $44.9 \pm 9.2 * *$ & $\begin{array}{c}42 \\
(25-67)\end{array}$ & $54.7 \pm 12.9^{* *}$ & $\begin{array}{c}51 \\
(28-79)\end{array}$ & $47.6 \pm 11.2$ & $\begin{array}{c}54 \\
(25-79)\end{array}$ \\
\hline Right ER $\left(^{\circ}\right)$ & $53.8 \pm 11.8^{*}$ & $\begin{array}{c}68 \\
(20-88)\end{array}$ & $49.1 \pm 11.8 *$ & $\begin{array}{c}52.7 \\
(24-76.7)\end{array}$ & $52.5 \pm 12.0$ & $\begin{array}{c}68 \\
(20-88)\end{array}$ \\
\hline Left ToHiRo $\left(^{\circ}\right)$ & $98.2 \pm 17.7$ & $\begin{array}{c}97 \\
(55-152)\end{array}$ & $102.9 \pm 17.1$ & $\begin{array}{c}73.7 \\
(62-135.7)\end{array}$ & $99.5 \pm 17.7$ & $\begin{array}{c}97 \\
(55-152)\end{array}$ \\
\hline Right ToHiRo $\left(^{\circ}\right)$ & $98.8 \pm 16.1$ & $\begin{array}{c}69.4 \\
(65-134.4)\end{array}$ & $103.8 \pm 17.5$ & $\begin{array}{c}76 \\
(58-134)\end{array}$ & $100.2 \pm 16.6$ & $\begin{array}{c}76.4 \\
(58-134.4)\end{array}$ \\
\hline ToHiRo Sum $\left(^{\circ}\right)$ & $197.0 \pm 33.0$ & $\begin{array}{c}154 \\
(120-274)\end{array}$ & $206.7 \pm 33.9$ & $\begin{array}{c}139 \\
(128-267)\end{array}$ & $199.7 \pm 33.5$ & $\begin{array}{c}154 \\
(120-274)\end{array}$ \\
\hline $\begin{array}{l}\text { Proportion of left ToHiRo } \\
\text { of ToHiRo sum }(\%)\end{array}$ & $49.8 \pm 1.8$ & $\begin{array}{c}10.3 \\
(45.8-56.1)\end{array}$ & $49.8 \pm 1.9$ & $\begin{array}{c}10.2 \\
(44.5-54.7)\end{array}$ & $49.8 \pm 1.8$ & $\begin{array}{c}11.6 \\
(44.5-56.1)\end{array}$ \\
\hline
\end{tabular}

IR: internal rotation; ER: external rotation; SD: standard deviation; ToHiRo: total hip rotation; ROM: range of motion. ${ }^{*} \mathrm{p}<0.05$, ${ }^{* *} \mathrm{p}<0.01$. The ROMs were measured with the participant in the prone position. 
of left-right difference in ToHiRo ROM differs depending on gender, no significant difference was found as the test statistic value was $0.118<$ rejection area $3.841(\mathrm{df}=1.0, \mathrm{p}=0.05)$. Therefore, the null hypothesis $\mathrm{H}_{0}$ could not be rejected and was put on hold (Table 4, Table 5).

On the greater side of the left and right ToHiRo, seven persons were 2 SD or more and two persons were 3 SD or more (Table 5).

Table 3. Distribution of total hip rotation range of motion

\begin{tabular}{|c|c|c|c|c|c|c|}
\hline \multirow{2}{*}{ ToHiRo ROM $\left(^{\circ}\right)$} & \multicolumn{2}{|c|}{ Male $(n=148)$} & \multicolumn{2}{|c|}{ Female $(n=57)$} & \multicolumn{2}{|c|}{ Total $(n=205)$} \\
\hline & Left ToHiRo & Right ToHiRo & Left ToHiRo & Right ToHiRo & Left ToHiRo & Right ToHiRo \\
\hline $50-59$ & 1 & 0 & 0 & 1 & 1 & 1 \\
\hline $60-69$ & 5 & 4 & 2 & 2 & 7 & 6 \\
\hline $70-79$ & 18 & 16 & 4 & 3 & 22 & 19 \\
\hline $80-89$ & 31 & 28 & 7 & 7 & 38 & 35 \\
\hline $90-99$ & 31 & 39 & 14 & 9 & 45 & 48 \\
\hline $100-109$ & 22 & 23 & 9 & 11 & 31 & 34 \\
\hline $110-119$ & 25 & 24 & 12 & 13 & 37 & 37 \\
\hline $120-129$ & 10 & 9 & 6 & 10 & 16 & 19 \\
\hline $130-139$ & 2 & 5 & 3 & 1 & 5 & 6 \\
\hline $140-149$ & 2 & 0 & 0 & 0 & 2 & 0 \\
\hline $150-159$ & 1 & 0 & 0 & 0 & 1 & 0 \\
\hline Total & 148 & 148 & 57 & 57 & 205 & 205 \\
\hline
\end{tabular}

ToHiRo: total hip rotaion; ROM: range of motion. The ROMs were measured with the participant in the prone position.

Table 4. Distribution of the proportion (\%) of the ToHiRo ROM on the side where the ToHiRo ROM was greater divided by the ToHiRo ROM sum

\begin{tabular}{ccccc}
\hline Class interval (\%) & \multicolumn{2}{c}{ Male $(\mathrm{n}=148)$} & \multicolumn{2}{c}{ Female $(\mathrm{n}=57)$} \\
\cline { 1 - 3 } from-under & Left Greater & Right Greater & Left Greater & Right Greater \\
& $\mathrm{n}(\%)$ & $\mathrm{n}(\%)$ & $10(40)$ & $\mathrm{n}(\%)$ \\
\hline $1-2$ & $24(39.3)$ & $34(39.1)$ & $10(40)$ & $8(46.9)$ \\
$2-3$ & $23(37.7)$ & $28(32.2)$ & $3(12)$ & $5(15.6)$ \\
$3-4$ & $8(13.1)$ & $19(21.8)$ & $0(0)$ & $2(6.3)$ \\
$4-5$ & $3(4.9)$ & $5(5.7)$ & $2(8)$ & $1(3.1)$ \\
$5-6$ & $1(1.6)$ & $1(1.1)$ & $0(0)$ & $1(3.1)$ \\
$6-7$ & $1(1.6)$ & $0(0)$ & $0(0)$ & $0(0)$ \\
$7-8$ & $1(1.6)$ & $0(0)$ & $25(100)$ & $32(100)$ \\
Subtotal & $61(100)$ & $87(100)$ & & \\
\hline
\end{tabular}

n: number of people; SD: standard deviation; ToHiRo: total hip rotaion; ROM: range of motion. The ROMs were measured with the participant in the prone position.

Table 5. Greater side of ToHiRo ROM classified by the standard deviation

\begin{tabular}{ccccc}
\hline Class interval & \multicolumn{2}{c}{ Male $(\mathrm{n}=148)$} & \multicolumn{2}{c}{ Female $(\mathrm{n}=57)$} \\
\cline { 1 - 1 } under & \multirow{2}{*}{$\begin{array}{c}\text { Left Greater } \\
\mathrm{n}(\%)\end{array}$} & $\begin{array}{c}\text { Right Greater } \\
\mathrm{n}(\%)\end{array}$ & $\begin{array}{c}\text { Left Greater } \\
\mathrm{n}(\%)\end{array}$ & $\begin{array}{c}\text { Right Greater } \\
\mathrm{n}(\%)\end{array}$ \\
\hline 1 SD & $56(64.4)$ & $44(72.1)$ & $23(71.9)$ & $19(76)$ \\
$2 \mathrm{SD}$ & $30(34.5)$ & $13(21.3)$ & $7(21.9)$ & $4(16)$ \\
$3 \mathrm{SD}$ & $1(1.1)$ & $2(3.3)$ & $2(6.3)$ & $2(8)$ \\
$4 \mathrm{SD}$ & $0(0)$ & $2(3.3)$ & $0(0)$ & $0(0)$ \\
Total & $87(100)$ & $61(100)$ & $32(100.1)$ & $25(100)$ \\
\hline
\end{tabular}

n: number of people; SD: standard deviation; ToHiRo: total hip rotaion; ROM: range of motion. The ROMs were measured with the participant in the prone position. 


\section{DISCUSSION}

We analyzed the existing data to clarify the hypothesis that there are left-right differences in ToHiRo ROM even among healthy people, and to show the distribution of the degree of left-right differences in ToHiRo ROM and whether or not there is a gender difference.

We found that the distribution of ToHiRo ROM according to the degree of left-right difference was significant even in normal participants. Since it became clear that there was no gender difference in the left and right differences in ToHiRo ROM, we decided to report the results together.

In this study, an important finding was that the ToHiRo ROM in healthy participants is widely distributed and that some healthy young people have significant left-right differences in the ToHiRo ROM.

The distributions of ToHiRo and ToHiRo ROM sum by class are shown in Tables 3 and 4, and the left-right difference of ToHiRo is analyzed by the ratio of ToHiRo on one side to ToHiRo ROM sum considering the flexibility of the joints (Tables 4 and 5).

Among them, the range of ToHiRo ROM was widely distributed from $55^{\circ}$ to $152^{\circ}$ (Tables 2 and 3 ), and the range of ToHiRo ROM sum was normally distributed in a wide range from $120^{\circ}$ to $274^{\circ}$ (Table 2 ).

From this wide distribution, it was found that there were participants with extremely small and extremely large hip joint rotation ROM. If the hip rotation ROM is extremely small, there is a possibility that they may have to compensate for the lack of movement in hip rotation ROM using the movement of the joint around the hip during various movements in daily life. Regarding the limits of ROM of the hip joint, there are reports that it is related to pain and osteoarthritis.

A previous study reported that the left ToHiRo ROM in the group with low back pain was smaller than that in the group without low back pain in Kendo athletes, and that the left-right difference in ToHiRo ROM was significantly larger in the group with low back pain than in the group without low back pain ${ }^{11)}$.

Another study comparing hip ROM of the judoka between the dominant and non-dominant legs reported that the group without low back pain had a larger ToHiRo ROM on the non-dominant side than the group with low back pain ${ }^{5)}$. One study reported that the limitation of knee flexion and extension and hip external rotation ROM were important determinants of disability in patients with osteoarthritis ${ }^{12}$. As described above, there is a report that the limitation of ROM of the hip joint is associated with low back pain and osteoarthritis. Therefore, the influence of the limitation of the ROM of the hip joint on the motor function in healthy participants could be an issue in the future.

There was an extremely large ROM in the distributions of ToHiRo ROM. If the ROM is extremely large, there is a possibility that there are two aspects of the joint. One is that flexibility is high and the other is that the joint does not have good stability.

Regarding the distribution of the left-right difference in the hip joint rotation ROM (Tables 4 and 5), it was found that there is a left-right difference that exceeds $2 \mathrm{SD}$ and $3 \mathrm{SD}$ even in young healthy people. It has been reported that asymmetry of hip joint rotation ROM and the reduction of ToHiRo are observed in patients with low back pain ${ }^{13)}$. It is intriguing that there are left-right differences both in healthy people and patients with low back pain. Regarding the therapeutic effect, there is a report that the fingertip-to-floor distance (FFD) was improved by passive hip internal and external rotation movements ${ }^{14)}$, and that knee pain was improved by adjusting the hip rotation $\mathrm{ROM}^{2}$.

Since the left-right difference in hip joint rotation ROM is associated with low back pain, knee pain, and FFD, research is needed on how the left-right difference in hip joint rotation ROM in normal participants is related to other physical characteristics and motor functions.

The proposed clinical application method is a modified version of the method described by Yokota et al ${ }^{15)}$. For example, when the left internal rotation ROM is $40^{\circ}$ and the left external rotation ROM is $50^{\circ}$, it becomes $40^{\circ}+50^{\circ}=90^{\circ}$. When $90^{\circ}$ of ToHiRo ROM is $100 \%$, the ratio of the left internal rotation range is $44.4 \%$. By setting the item "internal rotation ratio", it can be expressed as "left: $44.4 \% / 90^{\circ}$ ".

Notating the combined notations one by one on the left and right sides has the following advantage: understanding the balance between IR and ER on the same side, as well as the left and right side differences of ToHiRo, including the element of the difference in flexibility.

Future studies should examine how the left-right difference and flexibility of ToHiRo in healthy people are related to physical characteristics and motor function.

This study has some limitations. The reliability of the method of measuring the hip rotation ROM used in this study has not been investigated. We adopted a method that required four or more people for the measurement, but it seems difficult to apply this method to the clinic.

Presentation at a conference

A part of this research was presented at the Asian Confederation for Physical Therapy 2018, Cebu, Philippines on Nov 25th, 2018. 


\section{Funding}

This study was supported by the Department of Physical Therapy of International University of Health and Welfare.

\section{Conflict of interest}

There are no conflicts of interest to report.

\section{ACKNOWLEDGMENT}

We thank all participating students and faculty members for their cooperation.

\section{REFERENCES}

1) Ellison JB, Rose SJ, Sahrmann SA: Patterns of hip rotation range of motion: a comparison between healthy subjects and patients with low back pain. Phys Ther, 1990, 70: 537-541. [Medline] [CrossRef]

2) Cibulka MT, Threlkeld-Watkins J: Patellofemoral pain and asymmetrical hip rotation. Phys Ther, 2005, 85: 1201-1207. [Medline] [CrossRef]

3) Roach SM, San Juan JG, Suprak DN, et al.: Patellofemoral pain subjects exhibit decreased passive hip range of motion compared to controls. Int J Sports Phys Ther, 2014, 9: 468-475. [Medline]

4) Kotwicki T, Walczak A, Szulc A: Trunk rotation and hip joint range of rotation in adolescent girls with idiopathic scoliosis: does the "dinner plate" turn asymmetrically? Scoliosis, 2008, 3: 1. [Medline] [CrossRef]

5) Tak IJ, Weerink M, Barendrecht M: Judokas with low back pain have lower flexibility of the hip-spine complex: a case-control study. Phys Ther Sport, 2020, 45: 30-37. [Medline] [CrossRef]

6) Takeuchi K, Komatsu T, Tsukuda F: The influence of static stretching on the range of motion of internal and external hip rotation. Kobe International University Review, 2017, 109-114. (in Japanese).

7) Han H, Kubo A, Kurosawa K, et al.: Hip rotation range of motion in sitting and prone positions in healthy Japanese adults. J Phys Ther Sci, 2015, 27: 441-445. [Medline] [CrossRef]

8) Han H, Kubo A, Kurosawa K, et al.: Ipsilateral patterns of the rotational range of motion of the hip in healthy Japanese adults. J Phys Ther Sci, 2016, 28: 2550-2555. [Medline] [CrossRef]

9) Takemasa S, Shimada T: A consideration of ROM in young, adult and elderly men. ROM of the lower extremities and vertebrae in healthy men. Jpn J Phys Ther, 1990, 24: 492-495 (in Japanese).

10) David F, Robert P, Roger P, et al.: Statistics, 2nd ed. New York: W W Norton \& Co Inc, 1991. pp 475-492.

11) Kishi S, Morikita I: Range of motion of hip joints of male university kendo practitioners with lower back pain. J Phys Ther Sci, 2009, 21: 253-256. [CrossRef]

12) Steultjens MP, Dekker J, van Baar ME, et al.: Range of joint motion and disability in patients with osteoarthritis of the knee or hip. Rheumatology (Oxford), 2000, 39: 955-961. [Medline] [CrossRef]

13) Sadeghisani M, Manshadi FD, Kalantari KK, et al.: Correlation between hip rotation range-of-motion impairment and low back pain. A literature review. Ortop Traumatol Rehabil, 2015, 17: 455-462. [Medline] [CrossRef]

14) Nojima E, Sasaki M: Influences of the passive range of motion exercise of hip internal and external rotation on the finger-floor distance. Rigakuryoho Kagaku, 2005, 20: 187-190 (in Japanese). [CrossRef]

15) Yokota H, Nose T, Yoshioka K, et al.: Examination of the relationship between pelvic tilt angle and hip joint internal and external rotation range of motion during one-leg standing. J Jpn Phys Ther Assoc, 2011, 38: PI1-PI314 (in Japanese). 